\title{
Quaderni
}

QUADERNI Communication, technologies, pouvoir

68 | Hiver 2008-2009

Militantisme médical et fabrique des politiques de santé

\section{L'œuvre en actes : arts, médias et communications numériques}

Jean-Paul Fourmentraux

\section{(2) OpenEdition}

Journals

Édition électronique

URL : http://journals.openedition.org/quaderni/297

DOI : $10.4000 /$ quaderni.297

ISSN : 2105-2956

Éditeur

Les éditions de la Maison des sciences de l'Homme

Édition imprimée

Date de publication : 5 janvier 2009

Pagination : 95-101

\section{Référence électronique}

Jean-Paul Fourmentraux, "L'œuvre en actes : arts, médias et communications numériques », Quaderni [En ligne], 68| Hiver 2008-2009, mis en ligne le 05 janvier 2012, consulté le 21 avril 2019. URL : http:// journals.openedition.org/quaderni/297; DOI : 10.4000/quaderni.297 


\section{Technique}

\section{l'œuvre en actes :}

arts, médias et communications numériques

\author{
Jean-Paul \\ Fourmentraux
}

\author{
Maître de conférences \\ Université Lille 3, \\ laboratoire GERIICO \\ Chercheur associé à \\ l'EHESS, Paris
}

$\mathrm{Au}$ carrefour de l'innovation technologique et de l'art contemporain, le Net art présente en effet des enjeux de création inédits : mutations du travail artistique, redéfinition des modes de production et de circulation des œuvres, outils et stratégies renouvelés de leur mise en public. Apparu dans la deuxième moitié des années 90, cet art prolonge une forme d'art médiologique qui s'origine dans les expérimentations pionnières de l'art vidéo des années 1970 et fait suite aux premières pièces d'art par ordinateur des années 1980. Pour le monde des arts, Internet constitue simultanément, et dès son apparition, le support technique, l'outil créatif et le dispositif social d'une nouvelle variété d'œuvres. En s'inscrivant dans cette articulation, les œuvres du Net art se manifestent dans la conception de dispositifs interactifs spécifiques, mais aussi dans la production de formes de vies en ligne, et de stratégies de communication en réseau. Une part importante du travail de création consiste notamment à acheminer l'œuvre vers son public potentiel, pour que celle-ci puisse être agie. Or l'analyse de cette mise en exposition du Net art révèle différentes stratégies et tactiques artistiques : de la prise de rendez-vous ponctuels, à la fidélisation, et jusqu'à l'instauration de contrats de service ou de maintenance de la réception.

\section{L'œuvre d'art comme dispositif}

Le Générateur Poïétique d'Olivier Auber ${ }^{1}$ présente une figure idéaletypique de ce rapport aux œuvres d'art orchestré par un dispositif élaboré de captation du public. Inspiré du «jeu de la vie » de Conway (1976) le Générateur Poïétique permet à un grand nombre de personnes d'interagir individuellement en temps réel sur 
une seule et même image collective. Il s'agit en effet d'un jeu graphique où chaque joueur qui se connecte dispose d'un petit carré juxtaposé à celui des autres joueurs. En plaçant ses pixels colorés dans l'espace qui lui est alloué, en les juxtaposant à ceux des joueurs connexes ou en faisant écho au dessin des autres joueurs, chacun contribue à la réalisation d'un dessin collectif qui est constamment en évolution. La participation à cette expérimentation collective en temps réel est orchestrée par un contrat très contraignant, nécessitant de la part des internautes une disponibilité et une implication importante. L'annonce de l'événement se fait par E-mail et engage une prise de rendez-vous ponctuelle visant à partager dans le temps et dans l'espace la réalisation d'une image collective. Le Générateur Poïétique permet ainsi à plusieurs individus de se connecter à un moment donné sur un site, dont le lieu et l'heure du rendez-vous avaient été préalablement fixés par courrier électronique. Chaque participant doit avoir, suivant les recommandations préalables de l'artiste, procédé au téléchargement, à l'installation et l'apprentissage d'un logiciel de dessin bitmap. Respectant l'heure du rendez-vous soigneusement consignée dans son agenda, l'internaute est invité à rejoindre d'autres participants anonymes dans l'objectif d'un dialogue interfacé. Le résultat est ici le processus de communication lui-même, à travers l'action collective de composer une image. Autrement dit, l'action de chacun, visible simultanément par l'ensemble des participants, y détermine l'état de l'image à un instant donné, comme résultat de l'action de tous. Le Générateur Poiétique laisse ainsi entrevoir deux mouvements dont le titre même indique très bien la voie : 1) l'œuvre se trouve moins dans l'image que dans le dispositif qui l'a fait exister ; 2) le processus collectif importe ici bien plus que le résultat. Il y a bien un générateur qui renvoie au dispositif de création au sens pragmatique du terme dont nous avons préalablement restitué le caractère polysémique. Mais ce générateur est poiétique, dans le sens où aménage bien une action artistique nouvellement partagée avec le public. C'est donc à l'articulation pragmatique de ces deux notions - dispositifs et poḯsis - qu'invite l'artiste Olivier Auber.

L'utilisation, aujourd'hui extrêmement répandue, du vocable de dispositif, mot-valise s'il en est, s'origine dans des domaines d'application - la police, l'armée, le droit-, ou de recherche scientifique et esthétique très variés. Le terme renvoie également à des pratiques sociales très diversifiées, en liaison à des contextes disciplinaires encore largement déconnectés les uns des autres². Il est toutefois très fréquent de rapporter l'origine du concept à l'interrogation inaugurale de Michel Foucault ${ }^{3}$, appliquée à l'analyse de nouveaux dispositifs sociotechniques de surveillance tel que le panoptique disciplinaire. Toutefois, si l'élaboration rigoureuse, minutieuse et remarquablement féconde du concept de dispositif défini par Foucault permet une mise en relief du travail des procédures et des technologies, ces dernières y étaient souvent envisagées négativement, comme des instruments d'aliénation, de contrôle social ou de pouvoir.

Quadrillage des espaces, occupation et découpage du temps, gestion des déplacements, surveillance de tous les moments, règlements tatillons, panoptique, investissement des corps, etc. Dispositifs disciplinaires, normalisation plutôt que répression. Il s'agit de montrer 
l'intrication du pouvoir avec un savoir «technique» spécifique : «construction de tableaux », «prescriptions de manœuvres », « imposition d'exercices », « objectivation», « fonctionnalité », « calculabilité », surveillance », observations », " mesures comparatives qui ont la norme pour référence », « mécanismes scientifico-disciplinaires $»$, etc. ${ }^{4}$

Ultérieurement, un autre usage du concept de dispositif est initié, dans le domaine musical, par Pierre Schaeffer ${ }^{5}$ pour qualifier l'ensemble de règles de fonctionnement propres à un produit médiatique : le dispositif télévisuel de recherche y est par exemple entendu non comme le genre d'une émission, ni le sujet ou la fausse bonne idée, mais quelque chose comme « un piège tendu à l'animal humain pour sa capture en vue d'observation $»^{6}$. Toutefois, si elle inclue désormais l'action des sujets nécessaire à la disposition du piège en amont et à son actualisation en aval, cette acception focalise à nouveau exclusivement l'attention sur le déterminisme technique de cette relation. Depuis lors, un mouvement important de revalorisation des techniques a été engagé par la réflexion philosophique et sociologique, dans l'objectif de mieux rendre compte des usages de ce concept et des incidences qui en découlent. À cet égard, divers courants de recherche ont pris pour objet l'articulation des faits techniques et sociaux : l'ethnométhodologie, l'anthropologie des sciences et des techniques ou la tentative médiologique sont de ceux-là. Au cœur de ce mouvement, le concept de dispositif ${ }^{7}$ semble rendre compte du fait qu'un nouveau rapport aux objets caractérise la société contemporaine, ou qu'un autre rapport avec le monde matériel ou objectal est possible, non plus sur le mode de l'instrumentation ou de l'aliénation, mais sur le mode de la fréquentation et du contact, voire du jeu. Il devient alors possible de montrer le déploiement des dispositifs au cours des pratiques concrètes, là où se joue la médiation non pas avec mais dans l'environnement technique ${ }^{8}$.

L'analyse doit alors passer par les mêmes médiations, allant des humains et des institutions aux cadres de la perception, aux éléments matériels, et jusqu'aux détails les plus précis des œuvres et de leur production : elle permet ainsi de franchir le fossé désastreux qui séparait analyses sociales des conditions de l'art et analyses esthétiques ou sémiotiques des fameuses « œuvres elles-mêmes »?.

Dans une telle perspective, les objets cessent d'être asociaux, ils deviennent des supports, des instruments et des médiations pour l'action, engagés au même titre que les humains dans le déploiement de l'œuvre d'art et dans le tissage des mondes où elle fait sens et circule. En conséquence, au-delà du caractère prioritairement technique du dispositif, l'attention est focalisée sur une seconde acception, que la définition cidessus pourra (par extension) englober, renvoyant simultanément aux configurations et relations réalisées entre les éléments matériels et humains. Les nouvelles technologies de l'information placent de ce point de vue le dispositif au centre d'une relation homme-machine ${ }^{10}$. Cette relation à en effet désormais pour particularité d'engager autant des humains que les outils techniques qui composent leur environnement (aménagé) et cadrent leurs manières de faire (conventionnelles). La notion de dispositif est articulée à de nouveaux concepts sociologiques, avec lesquels elle conju- 
gue une vision du social en acte et une conception délibérément pragmatique de la sociologie : elle emprunte aux concepts d'attachement ${ }^{11}$, de configuration ${ }^{12}$ et de mise en œuvre ${ }^{13}$, dans l'objectif de servir une appréhension analytique appariée de l'écriture et de l'action en et de l'art.

\section{Poïétique de l'œuvre d'art}

Le terme poïesis est également l'objet de multiples redéfinitions et usages - conceptuels autant que pragmatiques - qui l'ancrent dans divers contextes disciplinaires et écoles de pensée. Ses origines étymologiques grecques lui confèrent le sens premier de création ou de production, du verbe poiein (faire) qui recouvre des acceptions différentes - voire contradictoires - au sein des écrits philosophiques. La philosophie voyant dans la praxis et la poïésis deux formes d'action humaine fondamentalement distinctes : alors que dans la poiésis la finalité de l'œuvre technique est une œuvre indépendante de l'action, la finalité de la praxis se trouve dans l'acte lui-même, l'effet ne s'y distinguant pas de la cause qui le produit. Selon la distinction aristotélicienne, la praxis défini des dispositions à agir accompagnées de règles, les actions et la connaissance pratique (au sens de Kant) ; la poiésis définit des dispositions à produire accompagnée de règles, les objets et la connaissance poïétique. Par conséquent, la praxis (action au sens strict) renvoie davantage aux actes (politiques ou moraux) valant par euxmêmes, là où la poiésis (création) correspond au travail compris comme la production d'un bien ou d'un service qui porte par lui-même de la valeur. En ce sens, Kant montre que l'art, qu'il définit comme un mélange de travail et de jeu, doit être distingué du travail de l'artisan. C'est également en ce sens qu'Hannah Arendt ${ }^{14}$ proposera ensuite de distinguer l'activité productive entre œuvre (poḯsis) et travail (praxis) : la finalité de la poïétique restera extérieure à celui qui fabrique, la fin de la production étant séparable du producteur; la finalité de la praxis, au contraire, est interne à l'action dont elle ne peut être séparée, le fait de bien agir étant ici le but même de l'action. Ce qui spécifie l'œuvre vis-à-vis du travail est que la première est toute entière tendue vers une fin : elle est la réalisation d'une activité productive intentionnelle et signifiante, là ou le travail n'est qu'action et répétition d'une tâche récursive sans visée de clôture. Aux choses incessamment (re)fabriquées par le travail s'opposent les Euvres, produit et résultat d'un acte intentionnel inscrit dans le domaine de la poḯsis.

Mais la richesse de l'analyse aristotélicienne comme des travaux ultérieurs d'Hannah Arendt résulte principalement de la tentative de liaison entre œuvre et action : on y trouve en effet plus que de simples dichotomies binaires entre praxis et logos, entre praxis et theoria, entre prattein et poein ou entre praxis et poïesis. Si dans de nombreuses lectures modernes d'Aristote, ces concepts restent disjoints, d'autres recherches plus contemporaines ${ }^{15}$ soulignent au contraire le dialogisme caractéristique de la pensée grecque.

On ne peut donc pas se représenter séparément, d'une part, des activités humaines, des actions, des actes, des conduites, des productions, des créations, relevant uniquement d'une " praxis » parce que contenant en elles leurs propres fins et, d'autre part, des produits seulement instrumentalisés d'une "poïese», asservis à d'autres fins, extérieures. Il y a continuellement 
interférence entre ces différents ordres qui concourent à de telles réalisations. (Ardoino, 1994, p. 113).

La poiésis (ré)introduit ici l'idée de processus : en mettant l'accent sur la production artistique comme œuvre, elle redéfinit également l'acte le travail artistique. Le concept se trouve alors augmenté d'une perspective dynamique visant à rendre compte de l'histoire ou du devenir - au sens de la genèse - des œuvres d'art. La relecture des travaux de Paul Valéry amène de nouveaux éléments de compréhension de l'idée de pratique poḯtique. Dans ses «Cahiers ${ }^{16}$ » notamment, Paul Valéry (1871-1945) promeut une Poiésis ${ }^{17}$ en projet, un esprit de laboratoire qui sous-tend «d'une part, l'étude de l'invention et de la composition, le rôle du hasard, celui de la réflexion, celui de l'imitation, celui de la culture et du milieu; d'autre part, l'examen et l'analyse des techniques, procédés, instruments, matériaux, moyens et supports d'action » (1937). À l'écart des réifications du génie artistique et de l'art conçu comme une œuvre d'inspiration ontologique, Valéry suggère d'appeler poïétique la science (à venir) des processus de création. L'accent y est (re)porté sur les activités humaines aux prises ${ }^{18}$ avec les techniques : «tout est en présence, tout en échanges mutuels et modifications réciproques (...) une intelligence qui organise un savoir en s'organisant elle-même $» .^{19}$

Le Générateur Poiétique d'Olivier Auber peut offrir une figuration de cet entre-deux. En évitant de caractériser une fois pour toute l'œuvre, l'artiste et le public, suivant des rôles et des positions à priori, il permet la circulation des uns vers les autres. En ce sens, il génère des usages et des pratique $^{20}$ dans et autour de dispositifs artistiques qui font l'œuvre. La poïésis n'apparaît pas ici strictement séparée de l'action, elle en déploie au contraire les possibles en créant un espace d'interprétation (ou d'expérimentation) qui conduit à ce qu'une chose (un objet, une image, un mot) ne soit jamais donnée pour ce qu'elle est (isolément), mais pour ce qu'elle peut être (dans une relation). L'entre-deux du dispositif poïétique réintroduit en effet une certaine plasticité entre l'idée d'une structure ou d'un ordre homogène et l'approche réticulaire mettant en évidence le flux généralisé des ensembles complexes ouverts, plus proches de l'indifférencié ou du chaos. Et par conséquent, le dispositif Poiétique oscille entre l'idéalisation d'une production esthétique délimitée, attribuable à un auteur singulier, porteuse de sa facture et de sa sensibilité individuelle, et le produit des usages qu'elle génère, résultant de ses appropriations et expérimentations par autrui. Ces dernières pouvant travestir et altérer considérablement la forme et les significations initialement déposées par l'artiste. Le mouvement pragmatique montre ainsi que l'objet-œuvre n'est pas une entité pleine des intentions de son auteur qu'elle ne ferait que traduire, et son destinataire n'est plus le récepteur vide et passif de ses effets préfigurés qu'il ne ferait qu'épouser pour mieux en contempler l'équilibre ${ }^{21}$. À l'opposé, ce n'est pas plus le regardeur qui fait l'œuvre, envisagée comme une entité organique disposée par un créateur démiurge et désintéressé. Ces deux acceptions sont des visions idéalisées, qui ne peuvent surgir qu'a posteriori et qui ignorent les multiples activités et ajustements qui concourent à la construction collective d'une stabilité toujours éphémère des énoncés et des formes. 
N
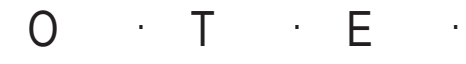

S

1. Olivier Auber, Générateur Poïétique, http://poieticgenerator.net; Olivier Auber, «Du générateur poḯtique à la perspective numérique », Revue d'esthétique 39 : 2001 ; Anne Cauquelin, 2006, Fréquenter les incorporels. Contribution à une théorie de l'art contemporain, Paris : PUF, p. 107.

2. Cf. la revue Hermès 25, « Le dispositif. Entre usage et concept », CNRS Éditions, 1999.

3. Qu'est-ce qu'un dispositif? Michel Foucault déploie ce questionnement dans son ouvrage: Foucault, M., Surveiller et punir. Naissance de la prison. Paris, Éditions Gallimard, 1975.

4. Cf. Berten, André., «Dispositif, médiation, créativité : petite généalogie », Hermès 25, 1999.

5. Alors responsable de l'ORTF en 1971.

6. Cf. Schaeffer, P., Machines à communiquer, Paris, Le Seuil, 1972, T. II, P.158-159. Repris dans Jacquinot-Delaunay, Monnoyer, Ibid, 1999, p. 10.

7. Sur l'application de la notion de «dispositif» aux arts technologiques, voir: Anne-Marie Duguet, «Dispositifs ». Communications $n^{\circ} 48$, Seuil, Paris, 1988, pp. 221-242; Jean-Paul Fourmentraux, Art et Internet. Les nouvelles figures de la création. Paris, CNRS Éditions, 2005.

8. Au sens d'Antoine Hennion, La passion musicale, une sociologie de la médiation, Paris, Métailié, 1993.

9. Hennion, A., 1993. «L'histoire de l'art: Leçons sur la médiation », Réseaux $n^{\circ} 60$, juillet-aout 1993.

10. Cf. Jacquinot-Delaunay, G., Monnoyer, L. (dir.1999). «Le dispositif. Entre usage et concept», Hermès, $\mathrm{n}^{\circ} 25$, Édition du CNRS, p. 10.

11. Cf. Bruno Latour, « Factures/ Fractures. From the concept of network to the concept of attachement», RES n³6, Automne 1999, pp. 20-31.
12. Cf. Anselm Strauss, La trame de la négociation. Ed. L'Harmattan, Paris, 1992.

13. Cf. Nelson Goodman, L'art en théorie et en action. Éditions de l'Éclat, Paris, 1996. Gérard Genette, L'œuvre de l'art. Tome 2. La relation esthétique, Seuil, Paris, 1996.

14. Arendt, H., La condition de l'homme moderne, Presses Pocket. 1983.

15. Voir notamment, Passeron, J-C., «Pour une approche poïétique de la création», Paris, Encyclopaedia Universalis, Symposium, Vol. I, 1991, pp.433/442. Ce dernier liera toutes les activités humaines de création, qu'elles soient d'art ou de science, à la «poïesis »: «La poïtique ? Est-elle d'ailleurs si nouvelle que cela? Sans remonter à Aristote, à Lessing, ..., sans parler de tous les artistes qui ont réfléchi sur leur travail, saluons P. Valéry, l'inventeur du mot, sinon de la chose. Obligé de se démarquer de la poétique, ... il propose la poïétique spécifique du faire : « le poïen, dont je veux m'occuper est celui qui s'achève en quelque œuvre et que je viendrai à restreindre bientôt à ce genre d'œuvres qu'on est convenu d'appeler œuvres de l'esprit » ».

16. C'est dans les Cahiers, aujourd'hui considérés comme le laboratoire expérimental de l'auteur, qu'episteme et poïsis sont en projet. Dans lesquels le but ultime de la recherche devient cette œuvre où les transformations des pensées paraîtraient plus importantes que toute pensée, où la recherche est envisagée comme un processus de vie mis en œuvre par le chercheur. Un corpus de 27000 pages en 29 volumes, constitué par des feuillets et des cahiers d'écolier sur lesquels Valéry a consigné de 1894 à 1945 sa méthode.

17. Cf. Patricia Signorile, Episteme et Poïésis, en projet...: ou l'esprit de laboratoire dans les Cahiers de Paul Valéry, In Rencontres MCX, «Pragmatique et complexité », 17 et 18 juin 1999. Internet : 
http://www.mcxapc.org/ateliers/21/doc8.htm. Voir également, Patricia Signorile, Paul Valéry, philosophe de l'art, Vrin, 1993.

18. La notion de « prise » à été proposée par Christian Bessy et Francis Chateauraynaud pour rendre compte, sur un mode actif, de la saisie perceptuelle et de l'estimation des objets d'art, Voir : Experts et faussaires. Pour une sociologie de la perception. Paris, Éditions Métailié, 1995. Sur la porosité de l'œuvre aux pratiques du public, voir: Etienne Souriau, 1956, « Du mode d'existence de l'œuvre à faire », in Bulletin de la Société Française de philosophie, 25 février 1956, p. 9 ; Umberto Eco, L'œuvre ouverte. Paris, Éditions Le Seuil, 1965.

19. Paul Valéry, Cahiers, éd. C.N.R.S., Vol. XIII, p. 273.

20. Selon Jacquinot-Delaunay (1999), « si les notions d'usage et de pratique sont souvent employées indifféremment cela ne devrait pas nous faire oublier que l'un est plus restrictif que l'autre: l'usage renvoie à la simple utilisation - fut-elle d'une machine complexe - tandis que la pratique intègre à cette dimension, les comportements, les attitudes et les représentations, voire les mythologies, suscités par l'emploi des techniques - dont la pratique Internet rend particulièrement bien compte à l'heure actuelle ». Cf. Jacquinot-Delaunay, G., Monnoyer, L. (dir.1999). «Le dispositif. Entre usage et concept». Hermès, ${ }^{\circ} 25$, Édition du CNRS, p. 10.

21. Cf. Hennion, A., Méadel, C. 1997, « Les ouvriers du désir. Du produit au consommateur, la médiation publicitaire », in Beaud, P., Flichy, P., Pasquier, D. et Quéré, L., (ed.), Sciences de la communication, Paris : Réseaux-Cnet, pp. 105-130.
$R \cdot E \cdot S \cdot U \cdot M \cdot E$

Les développements récents de l'art conçu par et pour Internet invitent à une redéfinition des formes de création collective, des dispositifs et des expériences médiatiques. En partant de l'analyse d'une œuvre qui a fait date - le Générateur Poïétique de l'artiste ingénieur Olivier Auber - cet article propose un retour réflexif sur ces notions : il éclaire la migration de leur signification, leurs usages renouvelés et leurs impacts sur les mondes de l'art contemporain. L'examen relie ainsi la pratique du Net art à l'histoire des innovations technologiques et ancre l'évolution de ses formes et de ses dynamiques à une approche pragmatique de la création contemporaine.

\section{Abstract}

Recent developments of art designed by and for Internet call for a redefinition of collective creation, devices and media experiences. Based on the analysis of a work which was a milestone - the Generator Poiétique of the artist-engineer Olivier Auber - this article offers a reflexive return on these concepts: it illuminates the migration of their meaning, their uses and their impacts renewed on the worlds of contemporary art. The review connects the practice of Net art in the history of technological innovations and its dynamics in a pragmatic approach of contemporary creation. 
